\title{
A CHARACTERIZATION OF HILBERT MODULES ${ }^{1}$
}

\author{
GEORGE R. GIELLIS
}

\begin{abstract}
For a proper $H^{*}$-algebra $A$, the relationship between *-representations of $A$ and Hilbert modules is discussed. It is shown that a Hilbert module structure can be defined on every essential*-representation of $A$.
\end{abstract}

1. Introduction. A Hilbert module is a right module $H$ over a proper $H^{*}$-algebra $A$, with a vector inner product $(\cdot, \cdot)$ mapping $H \times H$ into the trace class of $A$, where $(\cdot, \cdot)$ has certain properties analogous to a scalar valued inner product for a Hilbert space. P. P. Saworotnow [2] introduced the concept of a Hilbert module and showed that a linear space structure can be defined on $H$. Moreover, he proved that the vector inner product for $H$ generates a Hilbert space inner product for $H$.

Of course, the right module structure means that an element $a$ of $A$ can be represented as an operator $\pi(a)$ on $H$. The Hilbert space structure induced on $H$ is such that each $\pi(a)$ is linear with adjoint $\pi\left(a^{*}\right)$. In the terminology of [1], we have an essential ${ }^{*}$-representation of the $H^{*}$-algebra $A$ on the Hilbert space $H$, with the understanding that the representation is given by an antihomomorphism because of the right module structure.

The purpose of this paper is to show that the reverse process can be carried out. That is, given an essential *-representation of a proper $H^{*}$ algebra $A$ on a Hilbert space $H$, then a vector inner product can be defined for $H$ such that $H$ is a Hilbert module over $A$.

2. Definitions and notation. Throughout this paper $A$ will denote a proper $H^{*}$-algebra, with trace class $\tau(A)=\{a b \mid a, b \in A\}$. A projection in $A$ is a nonzero selfadjoint idempotent element of $A$. By an orthogonal projection base (OPB) for $A$ is meant a maximal family of mutually orthogonal projections in $A$. If $\left\{e_{\alpha}\right\}$ is an OPB, then $A=\oplus \sum_{\alpha} A e_{\alpha}=$ $\oplus \sum_{\alpha} e_{\alpha} A$.

Presented to the Society, September 3, 1971; received by the editors January 9, 1972.

AMS (MOS) subject classifications (1970). Primary 46H15, 46H25; Secondary 46K15, 46C10.

Key words and phrases. $H^{*}$ algebra, Hilbert module, vector inner product, ${ }^{*}$-representation.

${ }^{1}$ Part of this work is taken from the author's Ph.D. dissertation at the Catholic University of America, directed by Professor Parfeny P. Saworotnow. The research was partially supported by the Naval Academy Research Council.

(c) American Mathematical Society 1973 
It is shown in [3] that $\tau(A)$ is a 2-sided ideal in $A$. In fact, $\tau(A)$ is a Banach algebra with respect to the trace norm defined below. The trace functional on $\tau(A)$ is denoted as $\operatorname{tr}(\cdot)$. We have $\operatorname{tr}(a b)=\left[a, b^{*}\right]=$ $\sum_{\alpha}\left[a b e_{\alpha}, e_{\alpha}\right]$ where $[\cdot, \cdot]$ is the Hilbert space inner product for $A$ and $\left\{e_{\alpha}\right\}$ is an OPB. The trace norm $\tau(\cdot)$ on $\tau(A)$ is defined by $\tau(x)=\operatorname{tr}([x])$, where $[x]^{2}=x^{*} x$.

Definition. A right module $H$ over $A$ is called a Hilbert module if there exists a $\tau(A)$-valued function $(\cdot, \cdot)$ defined on $H \times H$ with the following properties:

(1) $(f+g, h)=(f, h)+(g, h)$ for all $f, g, h \in H$.

(2) $(f, g)^{*}=(g, f)$ for all $f, g \in H$.

(3) $(f, g a)=(f, g) a$ for all $f, g \in H$ and each $a \in A$.

(4) For each nonzero $f \in H$ there exists $a \neq 0$ in $A$ such that $(f, f)=a^{*} a$.

(5) $|\operatorname{tr}(f, g)|^{2} \leqq \tau(f, f) \tau(g, g)$ for all $f, g \in H$.

(6) $H$ is complete with respect to the metric

$$
d(f, g)=\{\operatorname{tr}((f-g, f-g))\}^{1 / 2}=\tau((f-g, f-g))^{1 / 2} .
$$

We can assume that a scalar multiplication is defined on $H$ such that $\lambda(f a)=(\lambda f) a=f(\lambda a)$ for all complex numbers $\lambda, f \in H$ and $a \in A$. The equation $[f, g]=\operatorname{tr}((g, f))(f, g \in H)$ defines the Hilbert space inner product referred to above. A detailed discussion of Hilbert modules may be found in [2].

\section{The characterization theorem.}

THEOREM. Let $K$ be a Hilbert space which is a right module over $A$ with the following properties:

(1) $f(\lambda a)=\lambda(f a)=(\lambda f) a$ for all complex numbers $\lambda, f \in K$ and $a \in A$.

(2) $[f a, g]=\left[f, g a^{*}\right]$ for all $f, g \in K$ and $a \in A$.

(3) If $f a=0$ for all $a \in A$ then $f=0$.

Then $K$ is a Hilbert module over $A$.

Remarks. Notice that if $K$ were a left module over $A$, the conditions of the theorem would be that $A$ have an essential*-representation on $K$. Since any *-representation of a Banach *-algebra is continuous (Theorem 4.1.20 of [1]), we know there exists a constant $m$ such that $\|f a\| \leqq$ $m\|f\||a|$ for all $f \in K$ and $a \in A$.

Lemma. Let $\left\{e_{\alpha}\right\}$ be an $O P B$ for $A$. Then $f=\sum_{\alpha} f e_{\alpha}$, for all $f \in K$.

Proof. Let $J$ be any finite set of indices $\alpha$. Then $f \rightarrow \sum_{\alpha \in J} f e_{\alpha}$ is clearly a projection operator on $K$, which implies that

$$
\left\|\sum_{\alpha \in J} f e_{\alpha}\right\|^{2}=\sum_{\alpha \in J}\left\|f e_{\alpha}\right\|^{2} \leqq\|f\|^{2} .
$$


Thus the series of orthogonal terms $f e_{\alpha}$ converges to an element $f^{\prime}$ of $K$. Since each $a \in A$ can be written as $a=\sum_{\alpha} e_{\alpha} a$, by continuity we have $f a=f^{\prime} a$ for all $a \in A$. Thus $f=f^{\prime}$.

Proof of THE Theorem. For $f \in K$, let $T_{f}$ denote the map $a \mapsto f a$. Then $T_{f}$ is a bounded operator from $A$ to $K$ and a simple calculation shows that $T_{f}^{*}(g a)=\left(T_{f}^{*} g\right) a$ for all $f, g \in K$ and $a \in A$. Let $b=T_{f}^{*}(f)$. Then $T_{f}^{*} T_{f}(x)=b x$ for all $x \in A$. This means that $b$ is a positive element of $A$. If $\left\{e_{\alpha}\right\}$ is an OPB for $A$, then $\sum_{\alpha}\left[b e_{\alpha}, e_{\alpha}\right]=\sum_{\alpha}\left[f e_{\alpha}, f e_{\alpha}\right]=\|f\|^{2}$. By Lemma 3 of [3], the convergence of the series implies that $b \in \tau(A)$. Since $b$ is a positive element of $\tau(A)$, there exists a selfadjoint $c \in A$, such that $c^{2}=b$.

We define a vector inner product for $K$ by the equation $(f, g)=T_{f}^{*}(g)$ $(f, g \in K)$. Since $T_{f}^{*}(g)$ can be expressed as a linear combination of four terms of the form $T_{h}^{*}(h)$, we have $(f, g) \in \tau(A)$. It is clear from the definition and the above discussion that conditions (1)-(4) of the Hilbert module definition are satisfied.

Finally, consider an OPB $\left\{e_{\alpha}\right\}$ for $A$. For $f, g \in K, \operatorname{tr}((f, g))=$ $\sum_{\alpha}\left[T_{f}^{*}(g) e_{\alpha}, e_{\alpha}\right]=\sum_{\alpha}\left[g e_{\alpha}, f e_{\alpha}\right]=[g, f]$. Thus conditions (5) and (6) are satisfied, since (5) is the Cauchy-Schwartz inequality and (6) requires completeness of the Hilbert space norm for $K$.

\section{REFERENCES}

1. C. E. Rickart, General theory of Banach algebras, University Series in Higher Math., Van Nostrand, Princeton, N.J., 1960. MR 22 \#5903.

2. P. P. Saworotnow, A generalized Hilbert space, Duke Math. J. 35 (1968), 191-197. MR 37 \#3333.

3. P. P. Saworotnow and J. C. Friedell, Trace-class for an arbitrary $H^{*}$-algebra, Proc. Amer. Math. Soc. 26 (1970), 95-100. MR 42 \#2304.

Department of Mathematics, United States Naval academy, Annapolis, MARYLAND 21402 